\title{
Interactions of fully nonlinear solitary wave with a freely floating vertical cylinder
}

\author{
B.Z. Zhou ${ }^{\mathrm{a}}$, G. X. Wu ${ }^{\mathrm{a}, \mathrm{b} *}$, Q.C. Meng ${ }^{\mathrm{b}}$ \\ ${ }^{a}$ College of Ship building Engineering, Harbin Engineering University, Harbin 150001, China \\ ${ }^{b}$ Permanentaddress: Department of Mechanical Engineering, University College London, Torrington Place, \\ London WC1E 7JE, UK
}

\begin{abstract}
Fully nonlinear numerical interaction of a transient wave with a three dimensional structure has been analysed based on a higher-order boundary element method (BEM). The BEM mesh on the free surface is generated through a combination of the structured and unstructured meshes. Through some auxiliary functions, the mutual dependence of fluid/structure motions is decoupled, which allows the body acceleration to be obtained without the knowledge of the pressure distribution. The solitary wave is used as the case study for the transient wave. It is obtained by the third order theory and the fully nonlinear theory. The accuracy of the present numerical model is verified through the steady propagation of a solitary wave and comparison with the published results for solitary wave interaction with a vertical wall. Simulations are then made to study solitary wave interaction with a truncated cylinder. Numerical results are provided for motions, forces and run-ups on the cylinder and comparison between results for the fixed cylinder and the freely floating cylinder is also made.
\end{abstract}

Keywords: Solitary wave; Transient wave-structure interaction; Fully nonlinear theory; Higher-order boundary element method

\section{Introduction}

For an offshore platform installed in the sea, it will encounter a variety of ocean waves. A good understanding of the mechanism of wave interaction with a structure is of vital importance for the safety of the personnel and the platform, the protection of the environment and investment. When the wave is small, which means that its amplitude is small relative to its length and the typical

${ }^{*}$ Correspondingauthor. Tel.: +44 2076793870; fax: +44 2073880180

E-mail address: g.wu@ucl.ac.uk 
dimension of the structure, the problem can be linearized in the simulation. In such a case, linear superposition can be used. An incoming wave can be decomposed into a various components. When the interactions of the structure with some typical wave components have been established, their combinations can then be used for a variety of waves. However, in large waves which in fact pose the major threat to the operation of the platform, the linear theory is no longer valid and superposition is no longer applicable. In such a case, each wave has to be considered separately.

There has been extensive work on a structure in nonlinear periodic waves, in particular the Stokes waves. Typical work includes Ferrant [1,2], Ferrant et al. [3], Ducrozet et al. [4], Zhou et al. [5] and Zhou \& Wu [6], where the total wave elevation and the total velocity potential are separated into two parts, based on the incoming wave from infinity and the disturbed potential by the body. The other type of work on the nonlinear problems in simulation is to follow the practice in the experiment in a physical tank. Wave is generated on one side while an artificial beach is installed on the other side. Typical work includes those by Ma et al. [7] , Wu \& Hu [8], Wang \& Wu [9] and Ma \& Yan [10] using the finite element method (FEM), and those by Liu et al. [11], Bai \& Eatock Taylor [12], Bai et al. [13], Yan \& Liu [14] based on the boundary element method (BEM).

The research on the nonlinear periodic waves has greatly improved our understanding of their interactions with a platform. A particular example is that the natural frequencies of tension-leg platform (TLP) and gravity-based platform (GBS) constructed from vertical cylinders are well above the dominant frequency of the wave. Although TLP and GBS with this kind of design avoid the linear resonance, resonance may be excited by higher order nonlinear force [6]. This can have serious implication to springing which usually refers to the resonant response of a platform in the stochastic sea state, when the stochastic properties of the motion have become steady. It is highly relevant to the fatigue analysis of the structure. A platform with such a design may also experience ringing, which is a transient response dominated by high frequency components. Ringing is more likely to be excited by a transient wave with a large peak or few peaks. It can last for a while even when the wave has well passed the structure [15-17].

The present work aims to shed some lights on how a nonlinear transient wave will interact with a floating structure. We choose the interaction between the solitary wave and a freely floating vertical cylinder as the example. The work can be readily extended to other types of transient waves and some realistic offshore structures [6]. There has been extensive research on propagation of the 
solitary wave, based on KdV equations with first order theory [18], second-order theory [19] and third order theory [20], as well as the fully nonlinear theory [21, 22]. There has also been extensive work on the reflection of a solitary wave by a vertical wall, which can be seen as a special case of solidary wave interaction with a structure. Most of them are carried out in the two dimensional. Maxworthy [23] and Chen \& Yeh [24] conducted experiments on it. Cooker et al. [25], Craig et al. [26] and Chambarel et al. [27] used BEM to solve the fully nonlinear equations, where the incoming wave is given by the fully nonlinear theory [21]. Su \& Mirie [28] carried out a perturbation analysis of two colliding solitary wave to the third order of accuracy. For the case of a fixed body, Sun et al. [29] used the first order solitary wave as the incident wave and studied its behaviour when passing a horizontal rectangular cylinder through the fully nonlinear simulation based on the FEM in two dimensional domains. For the three dimensional problems, Yates \& Wang [30] reported an experimental study on solitary waves scattered by a vertical cylinder. Mo et al. [31] employed the Euler equations to calculate the non-breaking solitary wave forces on slender piles. The governing equations were solved by the Finite Volume Method (FVM) and the free surface was tracked by the Volume of Fluid (VOF) method. Zhao et al. [32] carried out numerical simulations of the solitary wave scattered by a circular cylinder group based on the generalized Bousinesq equations together the FEM. Zhong \& Wang [33, 34] used the FEM to investigate the problem of solitary wave interaction with cylindrical structures. Cao \& Wan [35] took into account the viscosity of the fluid and used the Reynolds-Average Navier-Stokes (RANS) model. The first order solitary wave was used as the incoming wave for the above simulations apart from that by Mo et al. [31], where the second order solitary wave was adopted. Isaacson [36] considered the case of a free floating cylinder and the first order solitary wave was used as the incident wave. However only vertical motion was considered and limited data were provided.

In the above work, the cylinder is fixed apart from that in Isaacson [36]. The present work aims to provide new some insight into this type of interaction based on the fully nonlinear solitary wave and with extensive results. An initially vertical cylinder is placed in the wave and it will be free to respond to wave excitation and be set into motion which will further lead to wave radiation. The numerical model for this complete wave/body interaction process is based on a time-domain higher-order boundary element method [5]. The 4th-order Runge-kutta method is used for the time step marching on the free surface in the Lagrangian framework. By means of the auxiliary function 
method [37], the fully nonlinear mutual dependence of fluid flow and structure motions is resolved. The accuracy of the present numerical model is verified by comparing wave propagation of a solitary wave with the fully nonlinear solution from Clamond \& Dutykh [22]. Further comparison is made with the third order analytical solution and the published numerical results for the reflection of a solitary wave. Simulations are then made for solitary wave interaction with a truncated cylinder. The results are provided for motions, run-ups and forces on the cylinder.

\section{Mathematical model and numerical procedure}

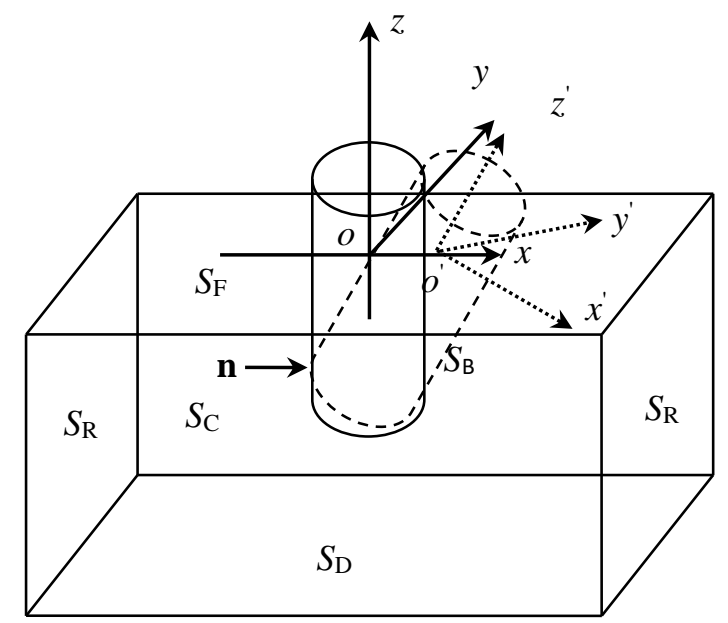

Fig. 1. Sketch of coordinate systems and computation domain

The problem of wave interaction with a vertical cylinder in water of depth $d$ is sketched in Fig. 1 . Two right-handed Cartesian coordinate systems are defined. One is the space-fixed system oxyz with the oxy plane on the undisturbed free surface and the $z$-axis pointing upwards. The other is a body-fixed system $o^{\prime} x^{\prime} y^{\prime} z^{\prime}$ with its origin $o^{\prime}$ placed at the centre of mass of the body. When the body is at its equilibrium position, these two sets of coordinate systems are parallel to each other. The centre of mass is located initially at $\mathbf{X}_{\mathrm{c} 0}$ in the space-fixed coordinate system, and $\mathbf{X}_{\mathrm{c}}\left(=\mathbf{X}_{\mathrm{c} 0}+\zeta\right)$ subsequently. Here $\zeta=\left(\zeta_{1}, \zeta_{2}, \zeta_{3}\right)$ is introduced to denote the translational displacements of the mass centre in the $x, y$ and $z$ directions respectively. The rotation of the body is defined through the usual Euler angles $\theta=(\alpha, \beta, \gamma)=\left(\zeta_{4}, \zeta_{5}, \zeta_{6}\right)$, to illustrate the displacements in roll, pitch and yaw, the terms commonly used in the naval architecture.

Based on the assumption that the fluid is ideal and incompressible, and flow is irrotational, the velocity potential $\phi(x, y, z, t)$ can be introduced, which satisfies the Laplace equation in the fluid domain $R$ 


$$
\nabla^{2} \phi=0
$$

It is subject to various conditions on the instantaneous boundary $S$ of the fluid domain, which includes the free surface $S_{\mathrm{F}}$, the body surface $S_{\mathrm{B}}$, the side surface $S_{\mathrm{C}}$ away from the body in the $y$ direction, the seabed surface $S_{\mathrm{D}}$ as well as the left and right side surface $S_{\mathrm{R}}$ away from the body in the $x$ direction. At $S_{\mathrm{C}}, S_{\mathrm{D}}$ and $S_{\mathrm{R}}$, the impermeable condition is given, that is $\partial \phi / \partial n=0$. On the free surface $S_{\mathrm{F}}$, the fully nonlinear kinematic and dynamic boundary conditions can be given in the following Lagrangian form

$$
\begin{gathered}
\frac{\mathrm{DX}}{\mathrm{D} t}=\nabla \phi \\
\frac{\mathrm{D} \phi}{\mathrm{D} t}=-g \eta+\frac{1}{2} \nabla \phi \cdot \nabla \phi
\end{gathered}
$$

where $g$ represents the acceleration due to gravity, $\mathbf{X}=(x, y, z)$ denotes the position vector of a fluid particle on the free surface, $\eta$ is the elevation of water surface measured from its mean level, $\frac{\mathrm{D}}{\mathrm{D} t}=\frac{\partial}{\partial t}+\mathbf{u} \cdot \nabla$ is the total derivative with $\mathbf{u}$ being the velocity of the fluid particle. The boundary condition on the body surface $S_{\mathrm{B}}$ is

$$
\frac{\partial \phi}{\partial n}=\mathbf{V} \cdot \boldsymbol{n}
$$

where $\mathbf{V}$ is the velocity of the body surface, $\mathbf{n}=\left(n_{x}, n_{y}, n_{z}\right)$ is the normal of the surface pointing out of the fluid domain, as shown in Fig. 1. The body surface velocity can be expanded as

$$
\mathbf{V}=\mathbf{U}+\boldsymbol{\Omega} \times \mathbf{r}_{\mathrm{b}}
$$

where $\mathbf{r}_{b}=\mathbf{X}^{\prime}$ is the position vector in the body-fixed coordinate system, $\mathbf{U}=\mathrm{d} \zeta / \mathrm{d} t=\left(U_{1}, U_{2}, U_{3}\right)$ is the translational velocity of the centre of mass, $\Omega=\left(U_{4}, U_{5}, U_{6}\right)$ is the rotational velocity, which can be linked to the temporal derivative of the Euler angles [38] .

The incoming wave considered here is the two dimensional solitary wave. Its lowest order is the solution of $\mathrm{KdV}$ equation, while the third order solution can be given as [20]

$$
\begin{gathered}
\eta / d=\varepsilon S^{2}-\frac{3}{4} \varepsilon^{2} S^{2} T^{2}+\varepsilon^{3}\left(\frac{5}{8} S^{2} T^{2}-\frac{101}{80} S^{4} T^{2}\right) \\
u / \sqrt{g d}=\varepsilon S^{2}+\varepsilon^{2}\left[-\frac{3}{4} S^{2}+S^{2} T^{2}+\left(1+\frac{z}{d}\right)^{2}\left(\frac{3}{4} S^{2}-\frac{9}{4} S^{2} T^{2}\right)\right] \\
+\varepsilon^{3}\left[\frac{21}{40} S^{2}-S^{2} T^{2}-\frac{6}{5} S^{4} T^{2}+\left(1+\frac{z}{d}\right)^{2}\left(-\frac{9}{4} S^{2}+\frac{15}{4} S^{2} T^{2}+\frac{15}{2} S^{4} T^{2}\right)\right. \\
\left.+\left(1+\frac{z}{d}\right)^{4}\left(\frac{3}{8} S^{2}-\frac{45}{16} S^{4} T^{2}\right)\right]
\end{gathered}
$$




$$
\begin{aligned}
w / \sqrt{g d} & =(3 \varepsilon)^{\frac{1}{2}}\left(1+\frac{z}{d}\right) S^{2} T\left\{\varepsilon+\varepsilon^{2}\left[-\frac{3}{8} S^{2}-2 S^{2}+\left(1+\frac{z}{d}\right)^{2}\left(-\frac{1}{2}+\frac{3}{2} S^{2}\right)\right]\right. \\
& +\varepsilon^{3}\left[-\frac{49}{640}-\frac{17}{20} S^{2}-\frac{18}{5} S^{4}+\left(1+\frac{z}{d}\right)^{2}\left(-\frac{13}{16}-\frac{25}{16} S^{2}+\frac{15}{2} S^{4}\right)\right. \\
& \left.\left.+\left(1+\frac{z}{d}\right)^{4}\left(-\frac{3}{40}+\frac{9}{8} S^{2}-\frac{27}{16} S^{4}\right)\right]\right\}
\end{aligned}
$$

where $\varepsilon=H / d$, with $H$ being the wave height, $S=\operatorname{sech}\left[\alpha\left(x-c t-x_{0}\right)\right]$ and $T=\tanh \left[\alpha\left(x-c t-x_{0}\right)\right]$. Other parameters are defined as

$$
\begin{aligned}
& c / \sqrt{g d}=1+\frac{1}{2} \varepsilon-\frac{3}{20} \varepsilon^{2}+\frac{3}{56} \varepsilon^{3} \\
& \alpha=\sqrt{\frac{3}{4}} \varepsilon\left(1-\frac{5}{8} \varepsilon+\frac{71}{128} \varepsilon^{2}\right)
\end{aligned}
$$

where $c$ is the phase velocity of the wave. The fully nonlinear solution of the solitary wave can be obtained from Clamond \& Dutykh [22].

The solution of the solitary wave is used at far upstream of the body as the boundary condition, where the disturbed potential can be assumed to have not arrived or have been absorbed by the numerical damping. To specify the initial condition, the cylinder is assumed to put into the wave suddenly and the potential on the free surface is assumed to be equal to the incident potential which can be obtained from integrating the velocity of the solitary wave solution along the free surface. We can start from a point at $x \ll x_{0}$, where the incident potential is almost zero. Let $l$ be the length along the free surface elevation in the $x-z$ plane, we then have

$$
\phi=\int_{0}^{l}\left(u \tau_{x}+w \tau_{z}\right) \mathrm{d} s
$$

at $t=0$ on the free surface. $\tau=\left(\tau_{x}, \tau_{z}\right)$ in the equation is the unit tangent vector the surface and can be obtained from

$$
\tau_{x}=\frac{1}{\sqrt{1+\eta_{x}^{2}}} ; \quad \tau_{z}=\frac{\eta_{x}}{\sqrt{1+\eta_{x}^{2}}}
$$

The governing Laplace equation in the fluid domain can be converted into an integral equation though the Green function $G$

$$
\alpha(\mathbf{p}) \phi(\mathbf{p})=\iint_{S}\left[\phi(\mathbf{q}) \frac{\partial G(\mathbf{p}, \mathbf{q})}{\partial n}-G(\mathbf{p}, \mathbf{q}) \frac{\partial \phi(\mathbf{q})}{\partial n}\right] \mathrm{d} s
$$

where $\mathbf{p}=\left(p_{x}, p_{y}, p_{z}\right)$ and $\mathbf{q}=\left(q_{x}, q_{y}, q_{z}\right)$ are source and field points, respectively, $\alpha(\mathbf{p})$ is the ratio of the solid angle to $4 \pi$. The whole boundary $S$ includes the free surface $S_{\mathrm{F}}$, the body surface $S_{\mathrm{B}}$, the 
seabed surface $S_{\mathrm{D}}$, the side surface $S_{\mathrm{C}}$ as well as the boundary at far upstream and downstream $S_{\mathrm{R}}$. At $S_{C}$ and $S_{R}$, we specify the normal derivative of the potential $\partial \phi / \partial n$ equal to zero, as they are taken sufficiently far away. For cases in which the computation domain is symmetric about the $y=0$ plane, and the seabed is horizontal, the potential due to a simple Rankine source and its image with respect to the symmetry plane $(y=0)$ and the seabed $(z=-d)$ can be chosen as the Green function, or

$$
G(\mathbf{p}, \mathbf{q})=-\frac{1}{4 \pi} \sum_{i=1}^{4} \frac{1}{R_{i}}
$$

where

$$
\left\{\begin{array}{l}
R_{1}=\sqrt{\left(q_{x}-p_{x}\right)^{2}+\left(q_{y}-p_{y}\right)^{2}+\left(q_{z}-p_{z}\right)^{2}} \\
R_{2}=\sqrt{\left(q_{x}-p_{x}\right)^{2}+\left(q_{y}+p_{y}\right)^{2}+\left(q_{z}-p_{z}\right)^{2}} \\
R_{3}=\sqrt{\left(q_{x}-p_{x}\right)^{2}+\left(q_{y}-p_{y}\right)^{2}+\left(q_{z}+p_{z}+2 d\right)^{2}} \\
R_{4}=\sqrt{\left(q_{x}-p_{x}\right)^{2}+\left(q_{y}+p_{y}\right)^{2}+\left(q_{z}+p_{z}+2 d\right)^{2}}
\end{array}\right.
$$

This removes $S_{\mathrm{D}}$ and $S_{\mathrm{C}}$ from Eq. (13) and the integral is performed only over the rest of surfaces with $y>0$.

The higher-order boundary element method is used to solve the boundary integral equation in Eq.(13) at each time step. The surface is discretized using the quadratic isoparametric elements. Through the shape functions $h_{k}(\xi, \varsigma), k=1, \ldots K$, in the local coordinate system $(\xi, \varsigma)$, one can write the position coordinates, the velocity potential and its normal derivative within an element in terms of their nodal values, in the following forms respectively:

$$
\mathbf{X}(\xi, \varsigma)=\sum_{k=1}^{K} h_{k}(\xi, \varsigma) \mathbf{X}_{k}, \quad \phi(\xi, \varsigma)=\sum_{k=1}^{K} h_{k}(\xi, \varsigma) \phi_{k}, \quad \frac{\partial \phi}{\partial n}=\sum_{k=1}^{K} h_{k}(\xi, \varsigma)\left(\frac{\partial \phi}{\partial n}\right)_{k}
$$

where $K$ is the total number of nodes of the element, and is equal to 8 and 6 for quadrilateral and triangular elements respectively.

The integral equation then becomes the following form: 


$$
\begin{aligned}
& \alpha(\mathbf{p}) \phi(\mathbf{p})-\sum_{i=1}^{N_{\mathrm{c}_{2}}} \int_{-1}^{1} \int_{-1}^{1} \sum_{k=1}^{K} \frac{\partial G(\mathbf{p}, \mathbf{q})}{\partial n} h_{k}(\xi, \varsigma) \phi_{k}|J(\xi, \varsigma)| \mathrm{d} \xi \mathrm{d} \varsigma \\
& +\sum_{i=1}^{N_{\mathrm{e}_{1}}} \int_{-1}^{1} \int_{-1}^{1} \sum_{k=1}^{K} G(\mathbf{p}, \mathbf{q}) h_{k}(\xi, \varsigma) \frac{\partial \phi_{k}}{\partial n}|J(\xi, \varsigma)| \mathrm{d} \xi \mathrm{d} \varsigma \\
& =-\sum_{i=1}^{N_{\mathrm{c}_{2}}} \int_{-1}^{1} \int_{-1}^{1} G(\mathbf{p}, \mathbf{q}) \frac{\partial \phi(\mathbf{q})}{\partial n}|J(\xi, \varsigma)| \mathrm{d} \xi \mathrm{d} \varsigma \\
& +\sum_{i=1}^{N_{\mathrm{e}_{1}}} \int_{-1}^{1} \int_{-1}^{1} \frac{\partial G(\mathbf{p}, \mathbf{q})}{\partial n} \phi(\mathbf{q})|J(\xi, \varsigma)| \mathrm{d} \xi \mathrm{d} \varsigma
\end{aligned}
$$

where $J(\xi, \varsigma)$ represents the Jacobian due to the transformation from the global system to the local one. $N_{\mathrm{e} 1}$ and $N_{\mathrm{e} 2}$ in the equation are the number of the discretized elements on the free surface $S_{\mathrm{F}}$ and solid surfaces including $S_{\mathrm{B}}, S_{\mathrm{C}}$ and $S_{\mathrm{R}}$. The above integrals are calculated numerically using the Gauss-Legendre integration method with 16 points for quadrilateral element and 4 points for triangular element if $\mathbf{p} \neq \mathbf{q}$. When $\mathbf{p}=\mathbf{q}$, point $\mathbf{q}$ is then linked to the nodal points of the element and the cell is subdivided. A polar coordinate transformation [36], which removes the singularity, is then employed to evaluate the integral within each sub element. The final matrix equation is obtained by imposing Eq. (17) at all the nodal points

$$
[A] \mathbf{x}=[B]
$$

in which $[X]$ includes the unknown normal velocities on the free surface and potentials on the solid surfaces, $[A]$ is the influence coefficient matrix and $[B]$ is a column obtained from the known terms of Eq. (18).

After solving the boundary value problem and obtaining the normal derivative of the velocity potential on the free surface, the free surface boundary condition in Eqs. (2) and (3) are used to advance $\mathbf{X}$ and $\phi$ in time. The three velocity components in the global system can be obtained from

$$
\left[\begin{array}{l}
\frac{\partial \phi}{\partial x} \\
\frac{\partial \phi}{\partial y} \\
\frac{\partial \phi}{\partial z}
\end{array}\right]=\left[\begin{array}{lll}
x_{\xi} & y_{\xi} & z_{\xi} \\
x_{\varsigma} & y_{\varsigma} & z_{\varsigma} \\
n_{x} & n_{y} & n_{z}
\end{array}\right]^{-1}\left[\begin{array}{c}
\frac{\partial \phi}{\partial \xi} \\
\frac{\partial \phi}{\partial \varsigma} \\
\frac{\partial \phi}{\partial n}
\end{array}\right]
$$

where $\partial \phi / \partial \xi$ and $\partial \phi / \partial \varsigma$ can be obtained from direct differentiation of Eq. (16) within each element. At a node shared by a number of elements, $\partial \phi / \partial \xi$ and $\partial \phi / \partial \varsigma$ at the node is obtained from the average of the results from these elements. The fourth order Runge-Kutta method [40] is used for time stepping in Eqs.(2) and (3). Therefore the boundary value problem is resolved at mini 
steps within each $\Delta t$.

The mesh on the free surface is generated based on the software Gambit at the beginning of the computation. In the Lagrangian form of the free surface boundary condition, the nodes of elements move both horizontally and vertically. It is possible that elements become distorted as time progresses. When the overall fluid boundary does not change significantly, mesh rearrangement on the free surface is implemented by adopting the spring analogy method to obtain the horizontal coordinates of new nodes on the free surface. Those nodes on the intersections with the body surface and the boundary are treated separately. Interpolation is then used to update the vertical coordinates as well as the potential at each new node. The details can be found in [5].

\section{Hydrodynamic forces and body motions}

The equation of motions for a rigid body can be written as [38]

$$
\begin{gathered}
{[M] \mathbf{U}=\mathbf{F}_{\mathrm{h}}+\mathbf{F}_{\mathrm{e}}} \\
{[I] \boldsymbol{\Omega}+\boldsymbol{\Omega} \times[I] \boldsymbol{\Omega}=\mathbf{N}_{\mathrm{h}}+\mathbf{N}_{\mathrm{e}}}
\end{gathered}
$$

where $\mathbf{U}$ and $\boldsymbol{\Omega}$ are defined after Eq.(5), and $\mathbf{U}=\left[a_{1}, a_{2}, a_{3}\right]^{\mathrm{T}}$ and $\boldsymbol{\Omega}=\left[a_{4}, a_{5}, a_{6}\right]^{\mathrm{T}}$ indicate the translational acceleration and the angular acceleration, respectively. $[M]$ and $[I]$ in the equations are mass and rotational inertia matrixes, and $\mathbf{F}_{\mathrm{e}}=\left[f_{\mathrm{e} 1}, f_{\mathrm{e} 2}, f_{\mathrm{e} 3}\right]^{\mathrm{T}}$ and $\mathbf{N}_{\mathrm{e}}=\left[f_{\mathrm{e} 4}, f_{\mathrm{e} 5}, f_{\mathrm{e} 6}\right]^{\mathrm{T}}$ are the external force and moment. As the rotational centre is at the centre of the body mass, there is no coupling between translational and rotational motions on the left hand sides of Eqs. (13) and (14). However the coupling does exist implicitly on the right hand sides. The hydrodynamic force $\mathbf{F}_{\mathrm{h}}=\left(f_{1}, f_{2}, f_{3}\right)$ and moment $\mathbf{N}_{\mathrm{h}}=\left(f_{4}, f_{5}, f_{6}\right)$ on the body can be obtained by integrating the pressure over its wetted surface

$$
f_{i}=-\rho \iint_{S_{\mathrm{B}}}\left(\frac{\partial \phi}{\partial t}+\frac{1}{2}|\nabla \phi|^{2}+g z\right) n_{i} \mathrm{~d} s
$$

where $\rho$ is the fluid density, $\left(n_{1}, n_{2}, n_{3}\right)=\mathbf{n}$, and $\left(n_{4}, n_{5}, n_{6}\right)=\mathbf{r}_{\mathrm{b}} \times \mathbf{n}$. If the incoming wave is only in the $x$ direction and the body is symmetric about the $x-z$ plane, $\mathbf{\Omega} \times[\mathbf{I}] \mathbf{\Omega}=0, \alpha=0, \gamma=0$.

An effective method for dealing with the temporal derivative in Eq. (22) is that proposed by Wu $\&$ Eatock Taylor [37]. In this approach, some auxiliary functions $\psi_{i}(i=1, \ldots, 6)$ are introduced. These functions satisfy the Laplace equation in the fluid domain. They are zero on the free surface and their normal derivatives $\partial \psi_{i} / \partial n=n_{i}$ on the body surface and are zero on the other rigid boundaries. Through the use of these functions, the equation of motion can be written as [37]

$$
\sum_{j=1}^{6}\left(m_{i, j}+c_{i, j}\right) a_{j}=Q_{i}-m_{33} g \delta_{i, 3}+f_{\mathrm{e} i}
$$


where

$$
\begin{gathered}
c_{i, j}=\rho \iint_{S_{\mathrm{B}}} \psi_{i} n_{j} \mathrm{~d} s \\
Q_{i}=\rho \iint_{S_{\mathrm{B}}}\left[\nabla \psi_{i}\left[\left(\mathbf{U}+\boldsymbol{\Omega} \times \mathbf{r}_{\mathrm{b}}\right) \cdot \mathbf{n}\right] \cdot\left[\nabla \phi-\left(\mathbf{U}+\boldsymbol{\Omega} \times \mathbf{r}_{\mathrm{b}}\right)\right]+\psi_{i}(\boldsymbol{\Omega} \times \mathbf{U}) \cdot \mathbf{n}\right] \mathrm{d} s \\
-\rho \iint_{S_{\mathrm{B}}+S_{\mathrm{F}}}\left(g z+\frac{1}{2}|\nabla \phi|^{2}\right) \frac{\partial \psi_{i}}{\partial n} \mathrm{~d} s
\end{gathered}
$$

where $m_{i, j}, m_{i+3, j+3}, i, j=1,2,3$ in Eq.(23) correspond respectively to $M_{i j}$ of $[M]$ in Eq.(20) and $I_{i j}$ of $[I]$ in equation (21) and $\delta_{i, j}=1(i=j)$ and $\delta_{i, j}=0(i \neq j)$. In this way the acceleration of the body can be obtained directly once the potential has been found without the knowledge of the pressure. By using these auxiliary functions, the nonlinear fluid-structure interaction problem is decoupled, and can be solved more easily.

\section{Numerical results}

We shall first verify our numerical model and calculation procedure through convergence study and comparison. In particular, when the initial solitary wave is given from the fully nonlinear theory [22], an accurate numerical solution should maintain the shape of the solitary wave as it propagates forward. This can be also used to verify the present procedure. After this, the reflection by a wall will be considered as a special case of solitary wave interaction with a fixed body and will be used for further comparison and verification. This is followed by solitary wave interaction with a fixed and vertical cylinder. Finally, its interaction with a freely floating cylinder will be considered. The water depth $d$, acceleration due to gravity $g$ and fluid density $\rho$ are used for nondimensionalisation. It means that the velocity is nondimensionalised by $(g d)^{1 / 2}$, time by $(d / g)^{1 / 2}$ and pressure by $\rho g d$. $\tau=t /(d / g)^{1 / 2}$ is introduced to represent the nondimensional time.

\subsection{Propagation of fully nonlinear solitary wave and its interaction with a wall}

The propagation of a single solitary wave is considered first for convergence study and comparison. The fully nonlinear solution by Clamond \& Dutykh [22] is used as the initial condition. The total length of the computational domain is taken as $L=35$. As the problem is in fact $2 \mathrm{D}$ in such a case, or it is independent of $y$, the computational domain is taken between $y=0$ and $y=W=0.4$. The initial wave crest is located at the origin of the space fixed coordinate system. The distance between the left inlet boundary and the origin of the coordinate system is $L_{\mathrm{left}}=15$, and that between the right 
outlet boundary and the origin is $L_{\text {right }}=20$.

The wave of height $\varepsilon=0.5$ is considered. Two different meshes are chosen for convergence study. In Mesh a, the element size is $\Delta x=\Delta y=0.4$ on the free surface. In the vertical direction of boundaries away from the body, three elements are specified and nodes are distributed uniformly. The element size of Mesh b is twice of that of Mesh a on the free surface and the solid boundary surface. The time step is selected as $\Delta \tau=0.04$ in both cases. Fig. 2 shows wave profiles at different times. It can be seen that the curves in Fig. 2 from two meshes are graphically identical as the wave propagates forwards. This shows that Mesh a can provide mesh converged results. Further convergence test is made with respect to time step using $\Delta \tau=0.04$ and $\Delta \tau=0.02$ respectively for Mesh a. The results are given in Fig. 3, and excellent convergence has once again been achieved. The combination of Figs. 2 and 3 shows that the results with Mesh a and time interval $\Delta \tau=0.04$ are of enough accuracy.

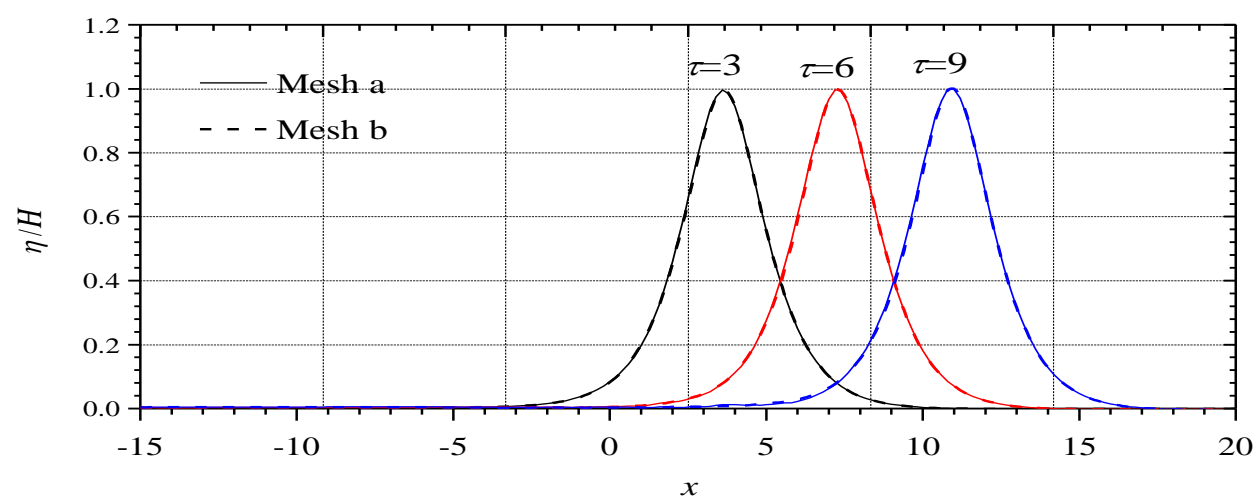

Fig. 2. Convergence study with mesh for the solitary wave of $\varepsilon=0.5(\Delta \tau=0.04)$

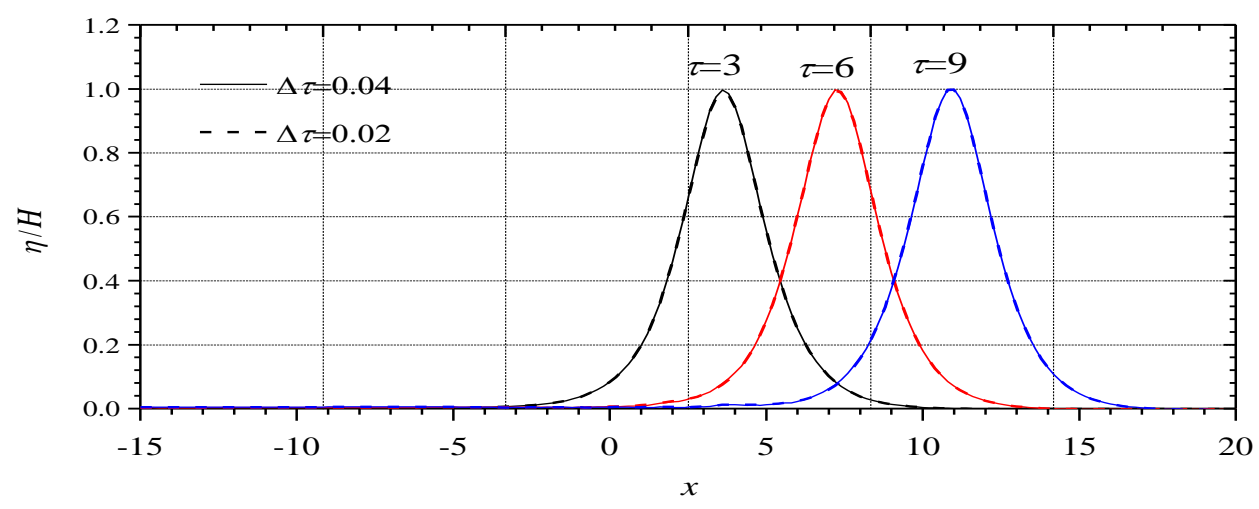

Fig.3. Convergence study with time step for the solitary wave of $\varepsilon=0.5$ (Mesh a)

After the convergence of the numerical results has been verified, we may investigate the effect of 
approximation in the initial solitary wave on the numerical results as time increases. If we use the fully nonlinear solution of Clamond \& Dutykh [22] as the initial condition in our present simulation, it can be expected the current results and those of Clamond \& Dutykh [22] should be numerically identical as the wave propagates forwards, as they are both based on the fully nonlinear theory. The third order solution of Grimshaw [20] is an approximation. It is sufficiently accurate for small wave height but its accuracy decreases as the wave height increases. If the third order solution is used as the initial condition in the present simulation, the numerical results should be close to the third order solution subsequently when $\varepsilon$ is small. However the difference is expected to appear when $\varepsilon$ increases. Two cases with $\varepsilon=0.2$ and 0.5 are calculated and results are given in Fig. 4. Fig. 4(a) shows that when $\varepsilon=0.2$ using the third order solution and that from Clamond \& Dutykh [22] as the initial condition respectively at $\tau=0$ does not make noticeable difference as $\tau$ increases. However it does lead to evident difference when $\varepsilon=0.5$, as shown in Fig.4 (b). When the solution of Clamond \& Dutykh [22] is used as the initial condition at $\tau=0$, the present simulation gives graphically identical result to that of Clamond \& Dutykh [22] as $\tau$ increases. However, when the third order solution is used as the initial condition at $\tau=0$, the wave height obtained from the present simulation decreases and the waveform becomes wider during propagation, and a dispersive tail can be observed behind the wave peak. This shows that accurate description of the incoming transient wave is highly important. When the solitary wave solution from the perturbation theory is used as the initial boundary conditions for the fully nonlinear analysis, as has been commonly adopted, some wave tails can appear due to numerical error. These tails can be eliminated when the solitary wave is obtained from the accurate fully nonlinear model. It is then further important to investigate whether the loss of its form will significantly affect the accuracy of the predicted loading on the structure.

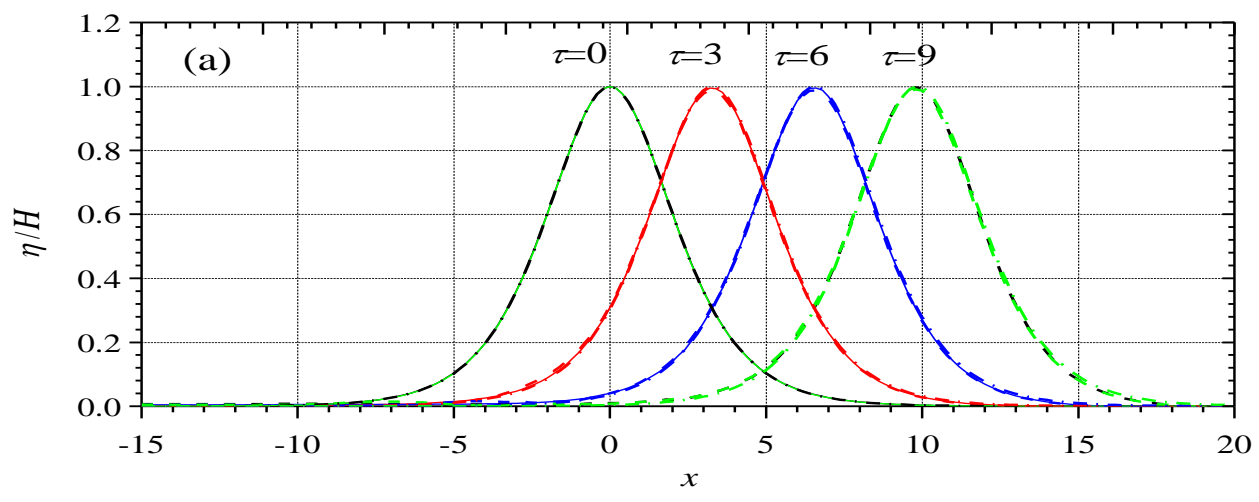




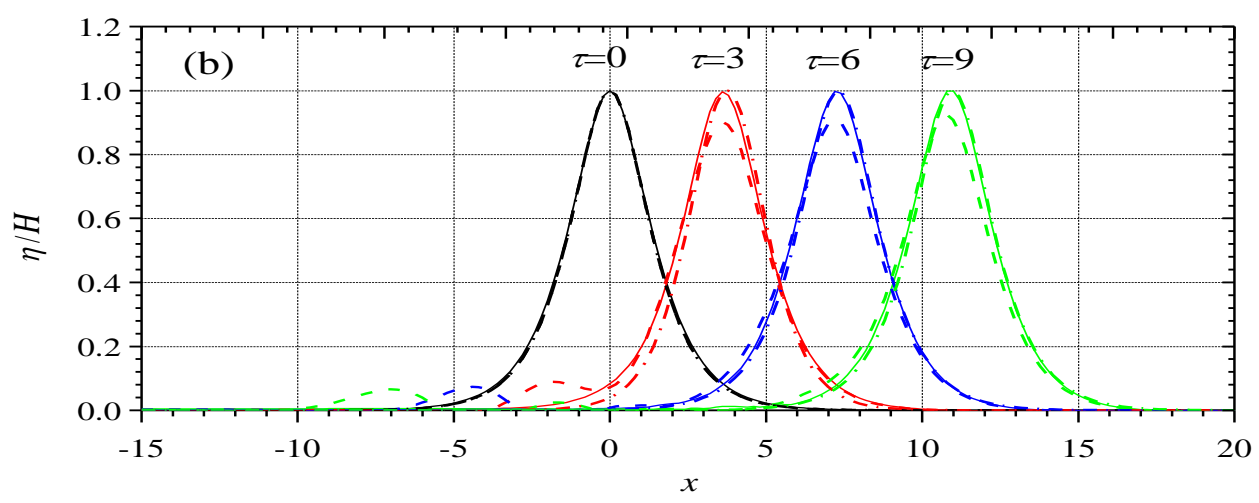

Fig.4. A comparison of free surface profiles. Solid lines are present result using the solution of Clamond \& Dutykh (2013) as the initial condition, dashed lines are present result using the third order solution as the initial condition and dash dotted lines are solutions of Clamond \& Dutykh [22]: (a) $\varepsilon=0.2$ and (b) $\varepsilon=0.5$

Wave reflection by a vertical wall has been extensively investigated and often used as a benchmark in the studies on wave-wave interactions. Here we consider the case with the solution of Clamond \& Dutykh [22] as the initial condition for further comparison. A vertical wall is suddenly placed at $x=15$ at $\tau=0$. After the convergence study with mesh, the element size is $\Delta x=\Delta y=0.3$ on the free surface. In the vertical direction of the wall, four uniform elements are specified. The time step is taken as $\Delta \tau=0.04$. Free surface profiles for incoming wave of height $\varepsilon=0.2$ and 0.5 are given in Figs. 5(a), (b), respectively. The times $\tau=13.9$ in Fig. 5(a) and $\tau=12.88$ in Fig. 5(b) are the moments of maximum run-up at the wall. It can be seen from Fig. 5(a) that at $\varepsilon=0.2$ the maximum run-up is about twice the wave height. At large time $\tau=25$, the wave has been reflected almost completely and it propagates in the opposite direction virtually in the original form. Fig. 5(b) shows that at $\varepsilon=0.5$ the reflected wave form is not the same as the original one at $\tau=25$. The peak is slightly lower and the there is a wave train behind the peak.

The maximum run-up is presented in Fig. 6 against the wave height $\varepsilon$. The fully nonlinear numerical results from Cooker et al. [25] and Chambarel et al. [27] and the third order analytical expression derived by Su \& Mire [29] are also included for comparison. It can be seen from the figure that the present results are in good agreement with the fully nonlinear numerical results of Cooker et al. [25] and Chambarel et al. [27]. Moreover, the third order analytical expression derived by $\mathrm{Su} \&$ Mire [29] gives results which are in excellent agreement with those from the numerical simulation when $\varepsilon<0.5$. 

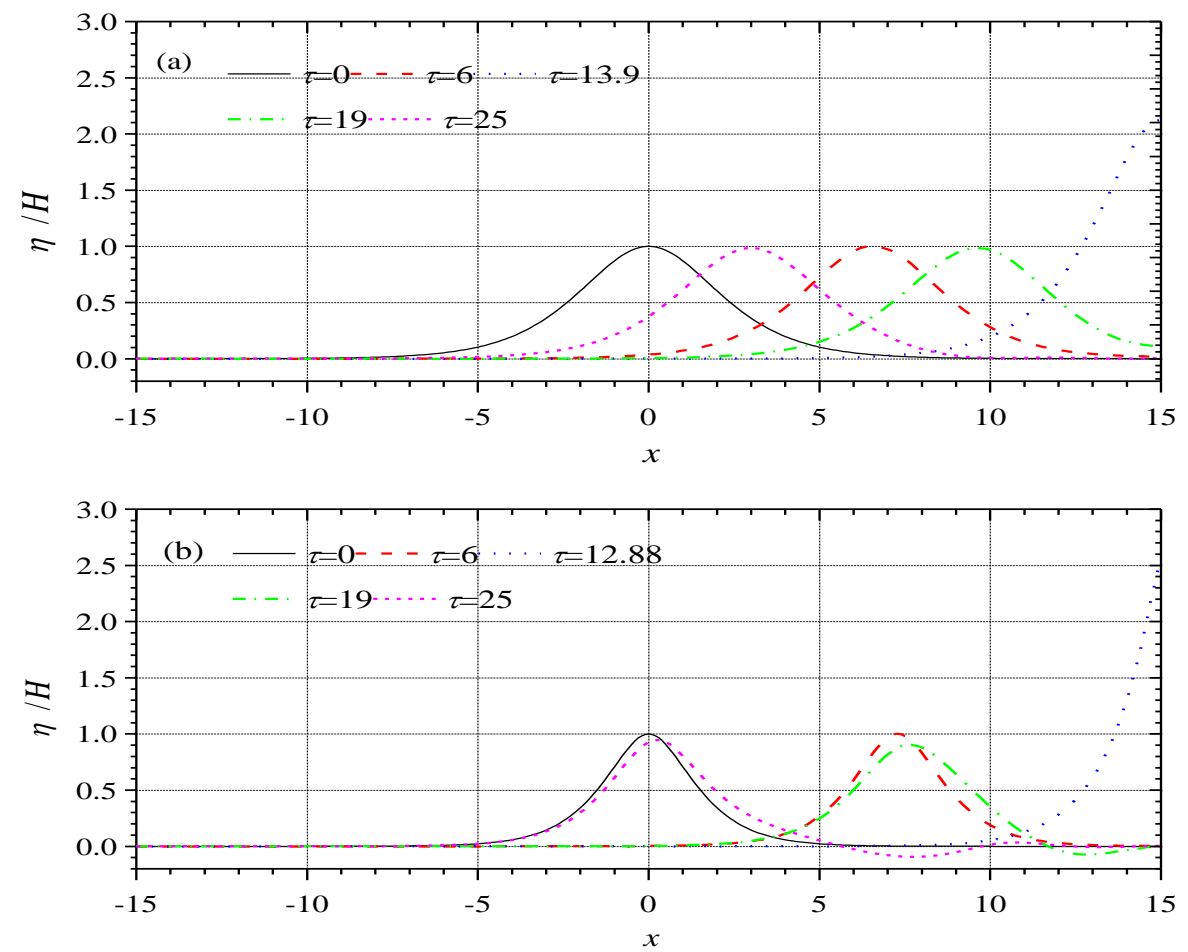

Fig. 5. Free surface profiles at different times for wave interaction with a wall at $x=15$ : (a) $\varepsilon=0.2$ and (b) $\varepsilon=0.5$

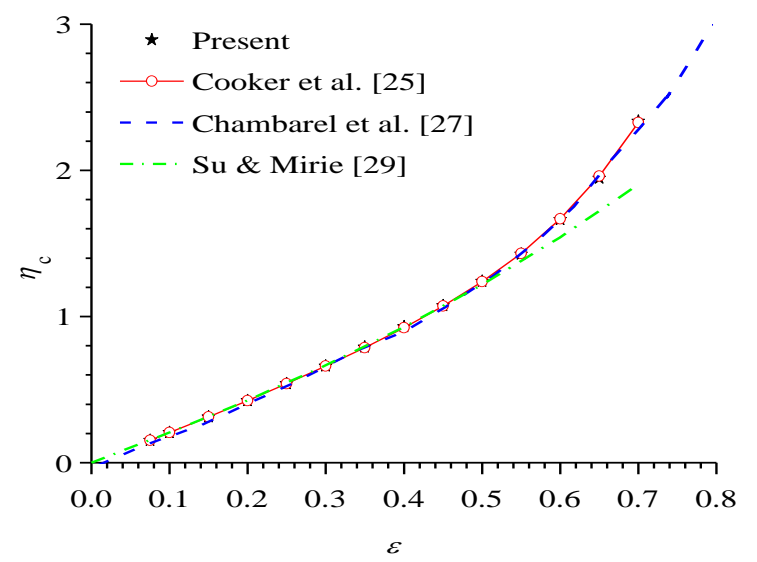

Fig.6. Maximum run-up at the wall as a function of incident wave height

Let $\tau_{0}$ be the time of maximum run-up at the wall. It has been observed in the numerical simulation that the wave crest with reflection effect lingers at the wall for a period of time $\tau_{\mathrm{r}}$. Let $\tau_{\mathrm{a}}$ and $\tau_{\mathrm{d}}$ be the attachment time and the detachment time respectively, at which the wave crest reaches and leaves the wall. Hence, the wall residence time is $\tau_{\mathrm{r}}=\tau_{\mathrm{d}}-\tau_{\mathrm{a}}$. The time $\tau_{0}$ at maximum run-up and the wall residence time $\tau_{\mathrm{r}}$ as a function of the incident wave height are presented in Fig. 7(a) and 7(b), respectively. Here, $\tau_{\mathrm{i}}$ in Fig. 7(a) is the time at which the peak of the undisturbed incident wave reaches the wall. The fully nonlinear numerical results from Cooker et al. [25] and Chambarel 
et al. [27], and the third order analytical expression derived by Su \& Mire [29] are also included for comparison. Numerical results for $\tau_{\mathrm{r}}$ are given only up to $\varepsilon=0.6$. As described by Chambarel et al. [27], collision could occur and a jet could be developed when $\varepsilon>0.6$. We in fact ran the case with $\varepsilon=0.65$ and it was observed that $\eta$ could be multivalued as time continues to progress and therefore the results are not included in the figure. It can be seen from the figure that the present results are in good agreement with the fully nonlinear one of Cooker et al. [25], while the third order solutions give the accurate results at much smaller wave height.
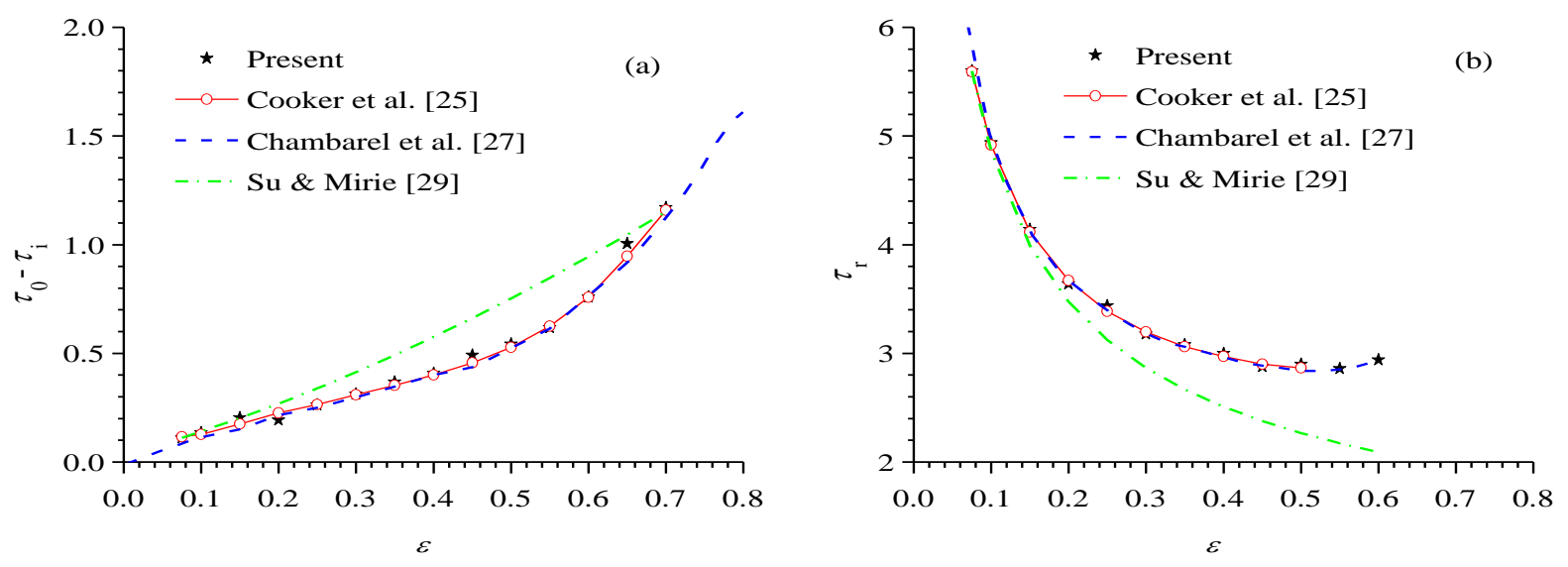

Fig.7. Time $\left(\tau_{0}-\tau_{\mathrm{i}}\right)$ at maximum run-up and wall residence time $\tau_{\mathrm{r}}$ as a function of incident wave height: (a) $\tau_{0}$ - $\tau_{\mathrm{i}}$;

(b) $\tau_{\mathrm{r}}$

\subsection{Solitary wave interaction with a truncated vertical cylinder}

The interaction of solitary wave with a vertical bottom-mounted cylinder has been studied experimentally by Yates \& Wang [30]. It has also been investigated numerically by Zhao et al. [32] and Cao \& Wan [35] using the first order solitary wave solution as the incoming wave. Here the fully nonlinear solution for the solitary wave will be used as the incident wave. A truncated cylinder of radius $R=1.5875$ and draught $B=0.5$ is placed in the fluid with its axis coinciding with the $z$ axis. The length of the computational domain is taken as $L=70$, while the half-width is $W=15.0$ to reduce the reflection of the radiated wave by the side boundary. The distance between the left inlet boundary and the axis of the cylinder is $L_{\mathrm{left}}=30$. The initial wave crest is placed at $x=-15.0$. Away from the body, the element size is taken as 0.4 in the horizontal direction. Twenty quadratic elements are used on the cylinder in the circumferential direction and four in the vertical direction. The time interval $\Delta \tau=0.04$ is used. 
The results for forces and moment acting on the cylinder for $\varepsilon=0.2$ and $\varepsilon=0.5$ from the numerical simulation with the fully nonlinear solution [22] as the incident solitary are shown in Figs 8 and 9, together with those from the incident wave obtained from the first order analytical solution [18] and the third order one [20]. It can be seen from Fig. 8 that there is hardly any obvious difference among the results at wave height $\varepsilon=0.2$. From Fig. 9 at $\varepsilon=0.5$, it can be seen that results from the first order and the third order solutions as the incoming wave underestimate the maximum forces and moment compared with those with the fully nonlinear solution as the incoming wave. Apart from the peak force however, all the curves are close to each other. This means that at $\varepsilon=0.5$ although there is some noticeable change of the form of the solitary wave when the third order solution is used as the incident wave for the nonlinear solution (see Fig.4(b)), it seems that the loss of the form does not have major effect on the force apart from the peak value.

Fig. 10 shows the time histories of free surface elevations around the cylinder for the incident wave height $\varepsilon=0.5$, together with the incident wave itself [22]. It can be seen from the figure that the maximum wave run-up occurs on the front side of the cylinder $\left(\theta=0^{\circ}\right)$ and its peak is about twice the incident wave height. This is due to the blockage effect of the front of the body and the wave has to move up. Subsequently, because of the overshoot of the peak, the wave elevation falls down from a high point to below the mean water level. The blockage effect is reduced away from the front of the body and the overshoot of the wave elevation is smaller at $\theta=45^{\circ}$ and it virtually disappears at $\theta=90^{\circ}$ (on the side of the cylinder). It is interesting to see further towards the back side of the cylinder, at $\theta=135^{\circ}$, the peak of the total wave elevation is much smaller than that of the incident wave, while on the back side of the cylinder $\left(\theta=180^{\circ}\right)$, the wave elevation nearly follows that of the incident wave apart from a delay to reach the peak.

Fig. 11 shows the snapshots of free surface on the $x-z$ plane at different times from $\tau=8$ to $\tau=30$, together with the incoming wave. The free surface around the cylinder circumference at different $\theta$ is also projected onto the $y=0$ plane to show its elevation around the cylinder. It can be seen that the peak of the total wave approaches the cylinder at a speed very close to that of the undisturbed incoming wave, although it lags slightly behind due to the blockage effect of the cylinder. Fig.11(c) shows that when the wave peak passes the body, the scattered wave becomes quite evident and it is in the usual oscillatory form. As the wave peak moves away from the body, the scattered wave also propagates away and the amplitude of its oscillation decreases. From Fig. 11(f), it can be seen that 
the total wave has almost reverted back to its original shape of incident wave and the scattered wave is disappearing.

To visualize the interaction process, the three dimensional free surface profiles are shown in Fig. 12 from $\tau=8$ to $\tau=30$. It can be seen from Fig. 10(a) that the maximum wave run-up on the front side of the cylinder occurs near $\tau=12$, which is consistent with what can be observed in Fig. 12(b). As the wave peak passes the cylinder, the ring wave propagating away from the cylinder is evidently visible. It can be also seen from Fig. 12(f) that the total wave system is returning to the shape of the incident wave, reflecting the well-known stability of the solitary wave.

_ Incoming wave from the fully nonlinear solution - - - Incoming wave from the third order solution -.- Incoming wave from the first order solution
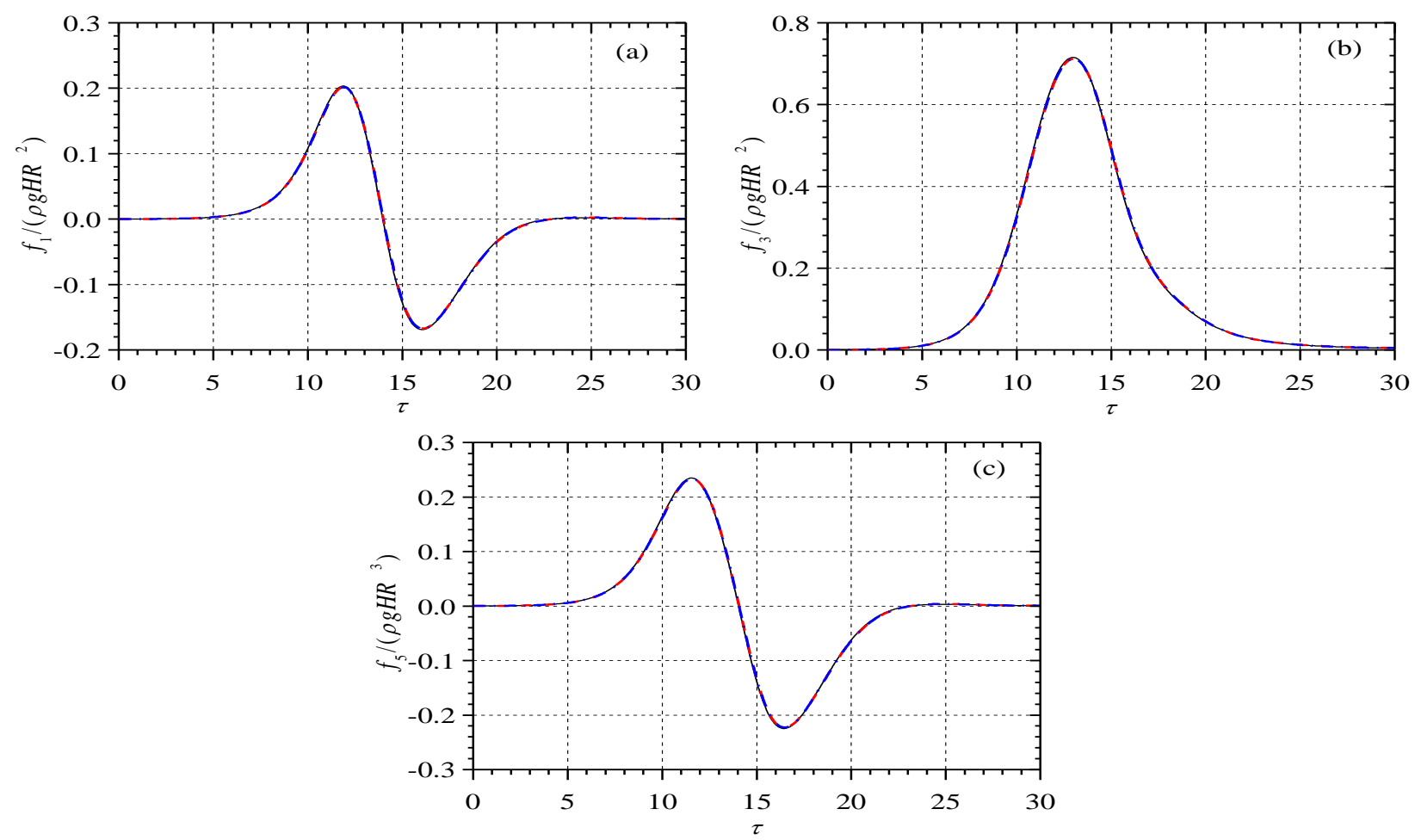

Fig. 8. Comparison of forces and moment acting on the cylinder for $\varepsilon=0.2$ between numerical simulations with the fully nonlinear solution and the third order solution as the incoming wave: (a) horizontal force; (b) vertical force and (c) moment about $y$-axis

_ Incoming wave from the fully nonlinear solution - - - Incoming wave from the third order solution -.. Incoming wave from the first order solution 

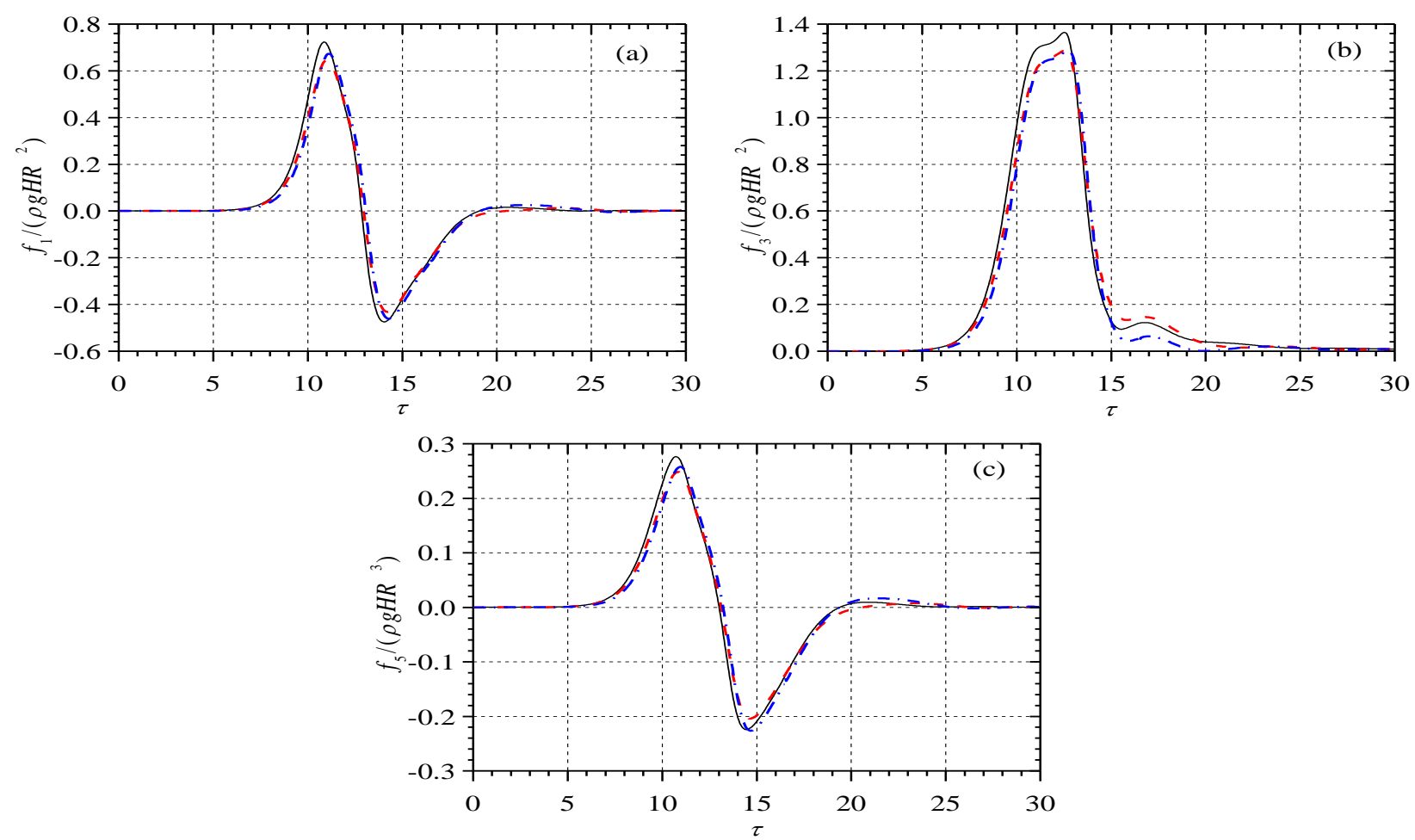

Fig. 9. Comparison of forces and moment acting on the cylinder for $\varepsilon=0.5$ between numerical simulations with the fully nonlinear solution and the third order solution as the incoming wave: (a) horizontal force; (b) Vertical force and (c) moment about $y$-axis
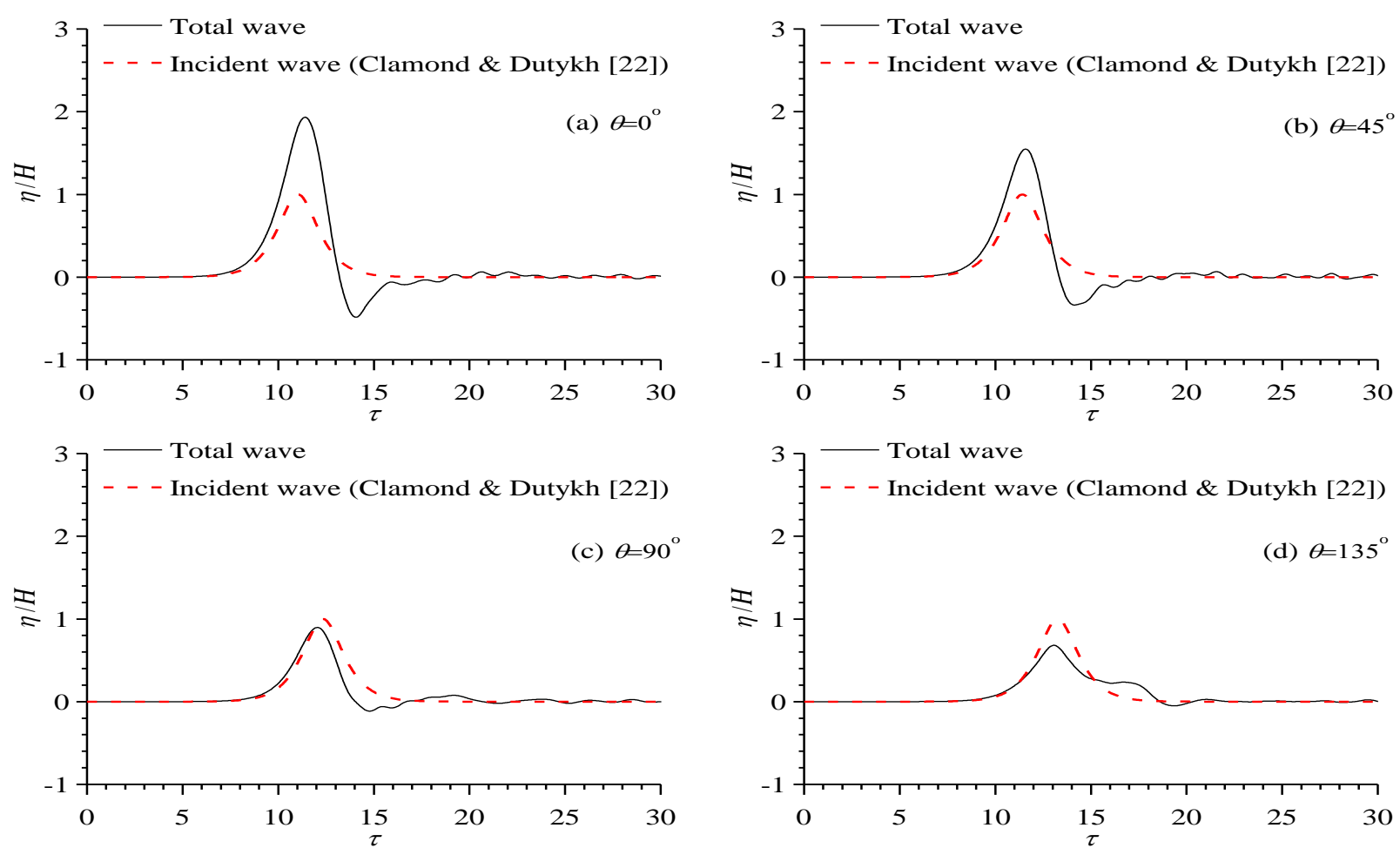


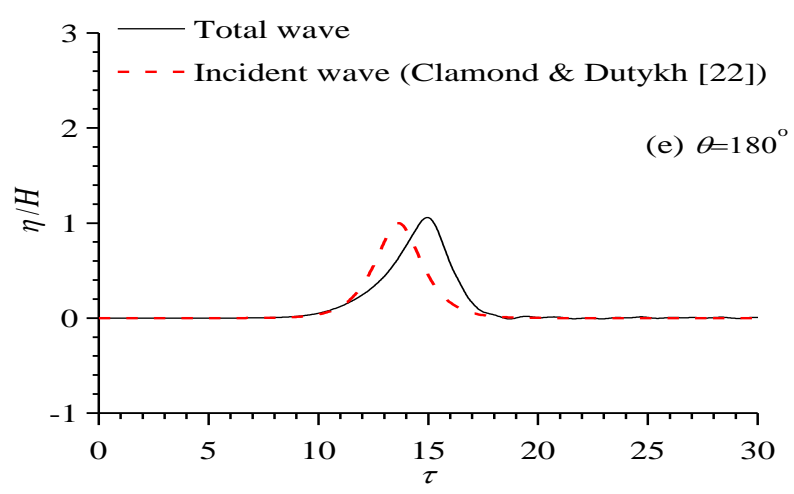

Fig. 10. Time history of free surface elevations around the cylinder for $\varepsilon=0.5 . \theta=0^{\circ}$ indicates the front side, and $\theta=180^{\circ}$ is the back side of the cylinder
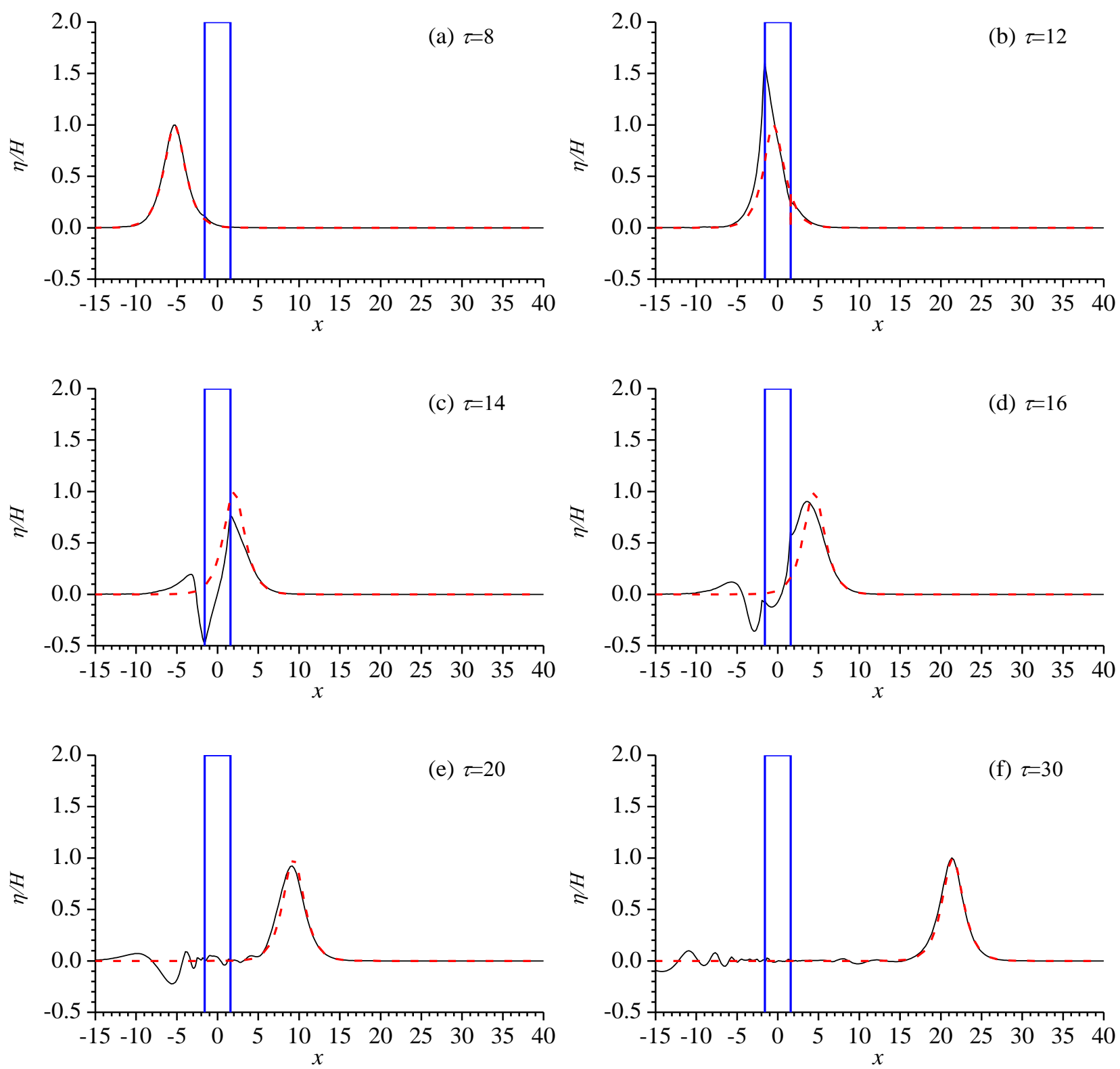

Fig.11. Free surface profile on the $x-y$ plane for $\varepsilon=0.5$, the dashed line represents the incident wave 
a

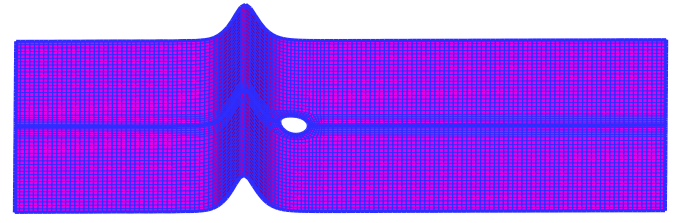

C

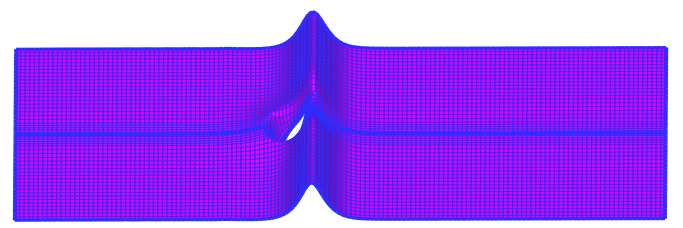

e

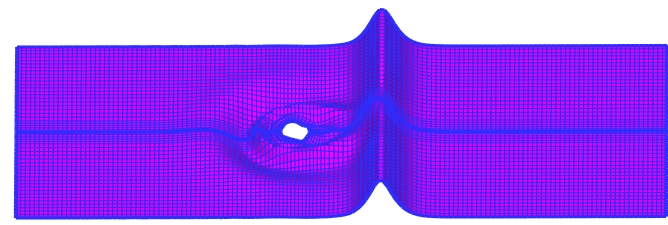

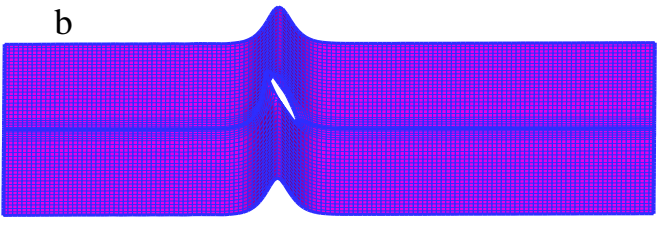
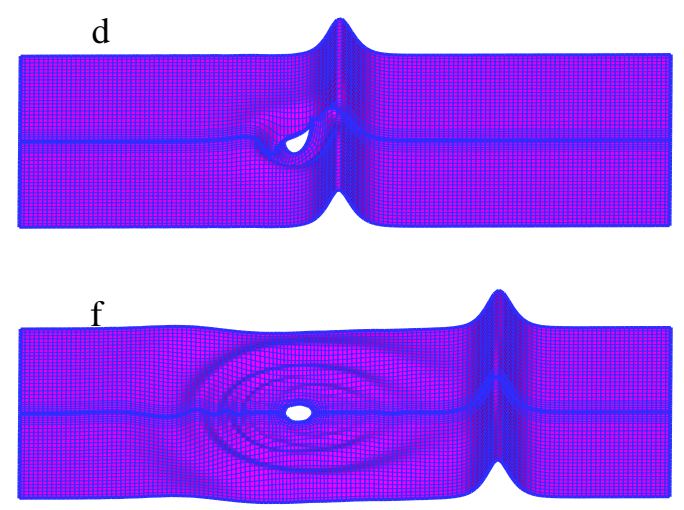

Fig. 12. Free surface evolution of solitary wave scattering by the cylinder for $\varepsilon=0.5$ : (a) $\tau=8$; (b) $\tau=12$; (c) $\tau=14$; (d) $\tau=16$; (e) $\tau=20$ and (f) $\tau=30$

Then, simulations will be made for the cylinder to be free to respond to the excitation to the solitary wave and be set into motion. As the incident wave considered here propagates only in the $x$ direction and the body is symmetric about $y=0$, it will respond only with motions in three degrees of freedom, namely translations in $x$ and $z$, as well as rotation about $y$. The mass of the body $m$ is defined as its initial displacement to guarantee that it is stationary initially, i.e., $m=3.9586$. The centre of the mass is located on the axis of the cylinder at $z=0.0$ when it is in the equilibrium position. The rotation inertial about $y^{\prime}$ is $I_{y}=2.5766$.

The translations in $x$ and $z$, as well as rotation about $y^{\prime}$ are shown in Fig. 13 for the incoming wave with $\varepsilon=0.4$. For the horizontal motion, a large drift motion can be seen due to the fact that there is no restoring force in this mode. In addition to the drift motion, a relatively small oscillatory motion is also visible in the $x$ direction, which is mainly caused by the oscillatory ring wave. In the vertical direction, the body is first lifted up with a relatively large peak when the wave peak arrives. Due to the fact that there is a restoring force in the vertical direction as a result of the difference in the weight and buoyancy, the motion undergoes several cycles of oscillation. The oscillatory motion is most obvious in the rotation about $y^{\prime}$. Although all the motions decrease with time as the solitary wave passes away, the motion in rotation is more persistent and its decay is at a much slower rate. This is because the disturbance of the rotation on the free surface is relatively small when the rotation centre is on the mean free surface. Thus the radiation damping coefficient in rotation about 
$y^{\prime}$ is relatively small compared with those in translations in the $x$ and $z$ directions, as has been noticed in [5]. As a result, the decay in rotation about $y^{\prime}$ is much slower than that in the other two modes.

The results for the forces and moment for the fixed cylinder and the moving cylinder are presented in Fig. 14. It can be seen that the loading in the case of moving body is smaller than that on the fixed body. That is because compared with the fixed body, the body response through motion will in general 'soften' the incoming wave excitation and as a result the wave loading is usually reduced. However, it should be noted that in some cases, the body motion and the incoming wave can be out of phase. The body can move towards the wave during its motion and the force for a freely floating cylinder can be bigger than that on the fixed cylinder. It can also be seen from Fig. 14(c) that the moment approaches zero faster after the wave has passed the fixed cylinder, while it oscillates for a longer period of time for the moving body because of the oscillatory rotational motion in Fig. 13(c).

The time histories of wave run-ups on the moving cylinder, which are effectively $z$ coordinates of the waterline at given $x^{\prime}$ and $y^{\prime}$, are shown in Fig. 15, together with those for the fixed cylinder and the incident wave elevation on the waterline itself. We notice that the latter two are in effect the $z$ coordinates at given $x$ and $y$. We also notice that the direction of $z$ axis is different from that of $z$. The run-ups in $z$ and $z$ ' directions are unified in the sense that they are all relative displacements along the body surface. Fig.15 (a) shows that the peak of the wave run-up on the front side of the moving body is much smaller than that of a fixed cylinder. This is due to the drift motion of the moving body, which has softened the blockage effect of the fixed cylinder. As $\tau$ increases, the wave run-up becomes negative and reaches a trough at approximately $\tau=13$. This is very much due to the vertical motion in Fig.13 (b), which has a peak around the same time. The oscillatory behaviour of the run-up is clearly linked to the oscillatory motions in the three degrees of freedom. The wave run-ups in other figures corresponding to different $\theta$ follow a similar pattern. It is roughly a combination of the wave run-up around a fixed cylinder in Fig.10 and the body motion of a moving a cylinder in Fig.13. 

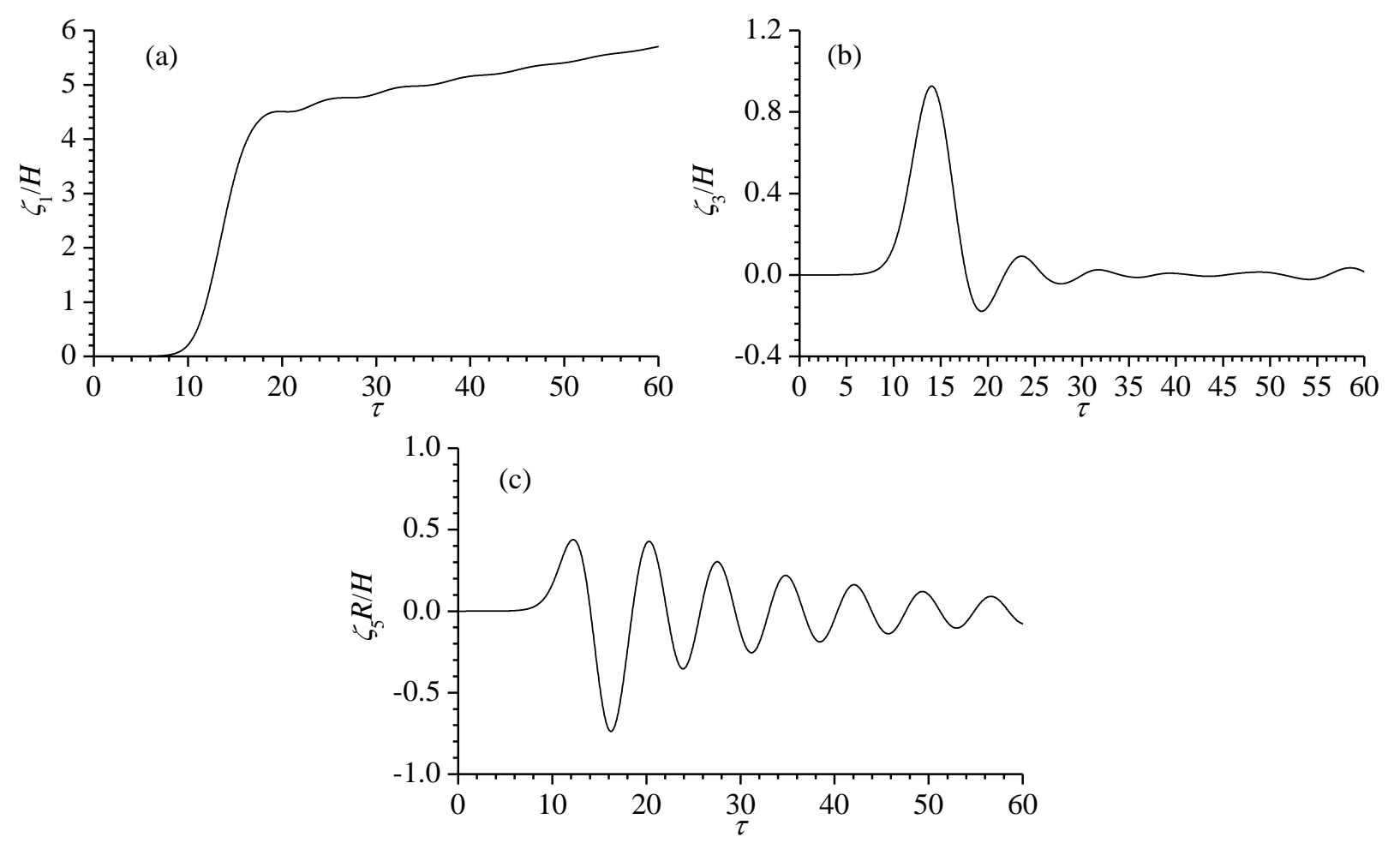

Fig. 13. Time history of motions of the floating truncated cylinder for $\varepsilon=0.4$ : (a) displacement in $x$ direction; (b) displacement in $z$ direction and (c) rotation displacement about $y^{\prime}$
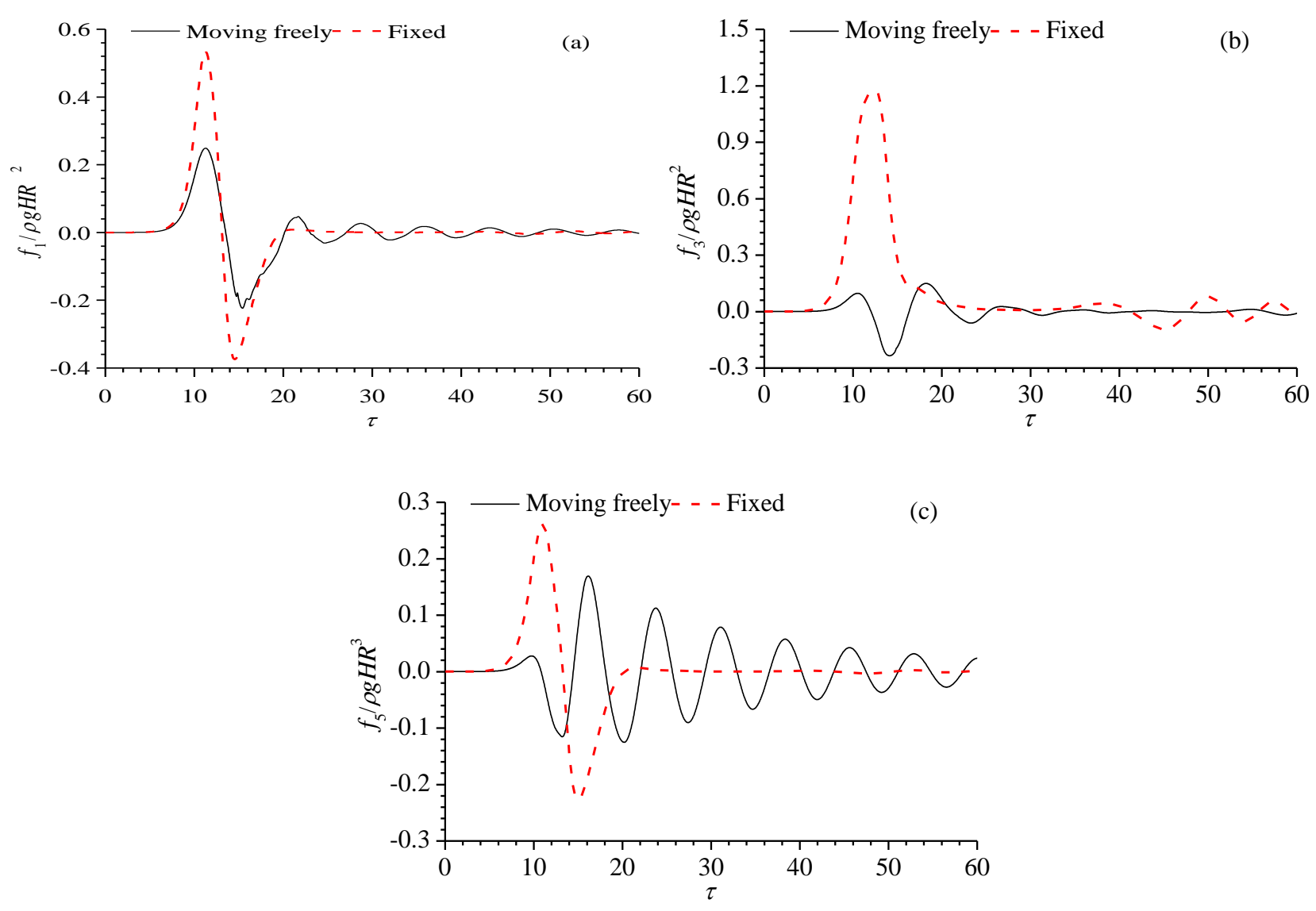

Fig. 14. Time history of wave forces and moment of the floating truncated cylinder for $\varepsilon=0.4$ : (a) horizontal 
force; (b) vertical force and (c) moment about $y$-axis
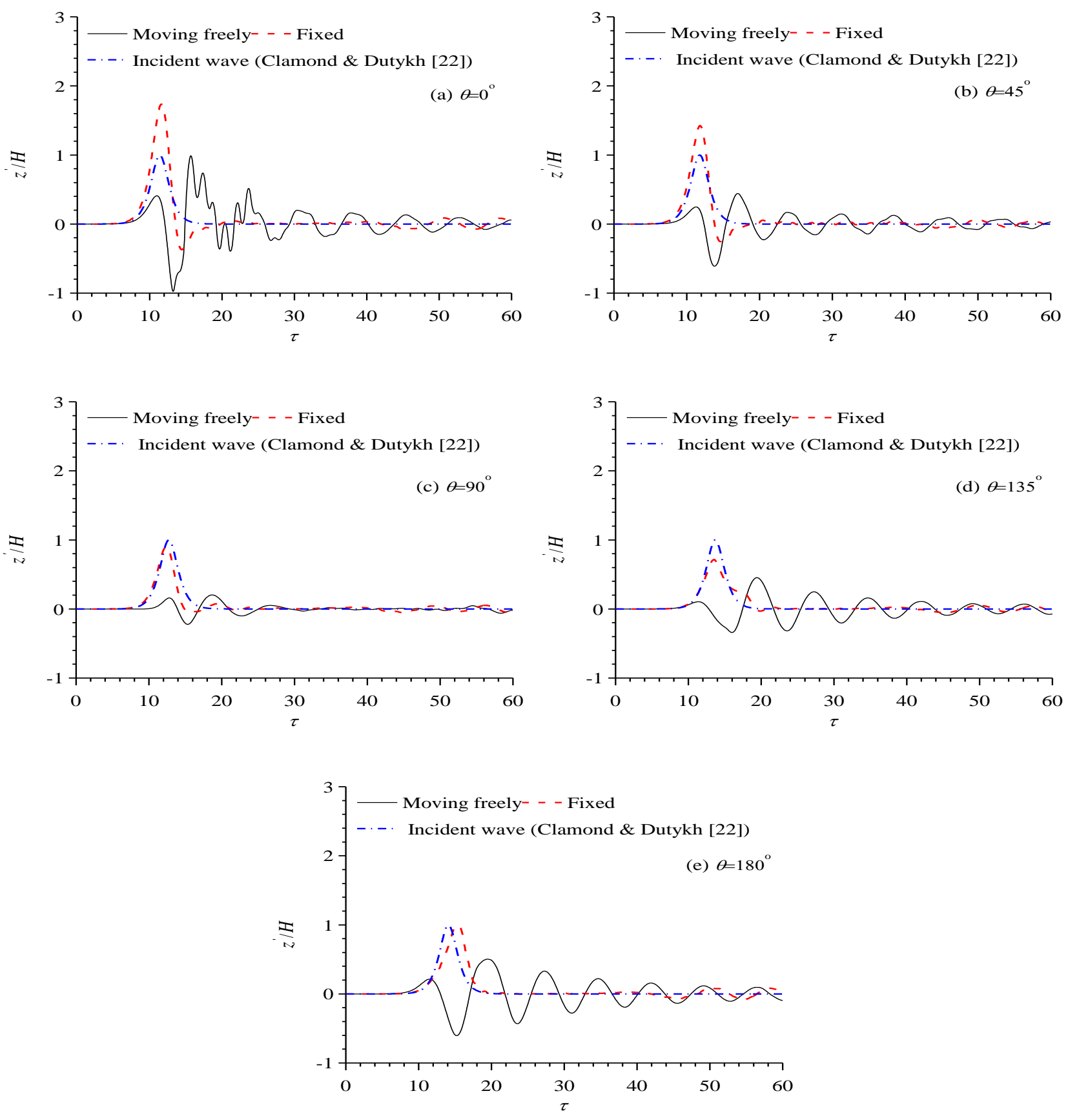

Fig. 15. Time history of free surface elevations around the cylinder for $\varepsilon=0.4 . \theta=0^{\circ}$ indicates the front side, and $\theta=180^{\circ}$ is the back side of the cylinder

\section{Conclusions}

A higher-order boundary element method has been developed to investigate the interactions of nonlinear transient wave and a three dimensional body. Quadratic boundary element method is used for solving the integration equation which is converted from the governing Laplace equation, 
together with the time-stepping procedure. The instantaneous wave elevations and potentials on the free surface are updated using the fourth-order Runge-Kutta method. The spring analogy method is used to redistribute the element nodes during the simulation to maintain the quality of the mesh without additional extensive computation requirement. The auxiliary function method is adopted to decouple the nonlinear mutual dependence of the fluid flow and structure motions, which makes it possible for the acceleration to be found before the detailed pressure distributions are known. The solitary wave, obtained from the third order theory and the fully nonlinear theory, is used as a case study for the transient wave. Extensive simulations have been made for the solitary wave and its interactions with a vertical cylinder, from which following conclusions can be made.

(1) At a smaller amplitude $\varepsilon=0.2$, the third order theory provides accurate solution for the solitary wave. When the third order solution is used as the initial condition and incoming wave, the solitary wave will maintain its form when propagating forward. At $\varepsilon=0.5$, however, the same exercise shows that when the third solution is used as the incident wave, its peak will be noticeably reduced and a wave tail can be observed behind the peak. The numerical solution of the solitary wave no longer maintains its form. However, it is subsequently found that the loss of its form does not affect the force and moment on a fixed vertical cylinder significantly apart from the peak value.

(2) When the solitary wave encounters a fixed cylinder, the peak of the wave run-up on the front side can be significantly higher than that of the incident wave, while it is about the same as the incident wave on the back side. For a moving cylinder, the behaviour of the wave run-up is almost due to a combination of that of a fixed cylinder and that caused by the motion the mean free surface relative to the moving body.

(3) When the solitary wave passes a freely floating truncated cylinder, it has a large drift motion in the horizontal motion due to zero restoring force. There is a large peak in the vertical motion when the peak of the solitary wave arrives, due to the buoyancy effect of the long wave. The rotational motion about the horizontal axis perpendicular to the wave direction and along the undisturbed free surface is persistent and decays very slowly even after the solitary wave has well cleared off the body. This is principally due to the relatively low radiation damping in this mode. This shows that the behaviour of the body in such a wave is very different from that in the periodic wave. The observed persistent oscillation is very similar to the behaviour of ringing which is persistent even when the wave has moved away, as has been observed in experiment and in field [15-17]. Thus the 
present work is an important step towards the analysis of a practical offshore platform in a variety of real transient waves.

\section{Acknowledgement}

The authors gratefully acknowledge the financial support from the Lloyd's Register Foundation (LRF) through the joint centre involving University College London, Shanghai Jiaotong University and Harbin Engineering University. The LRF helps to protect life and property by supporting engineering-related education, public engagement and the application of research.

This work is also supported by the National Natural Science Foundation of China (11472088, 51409066), the Open Fund of State Key Laboratory of Ocean Engineering of Shanghai Jiaotong University (1311).

\section{References}

[1] Ferrant P. Simulation of strongly nonlinear wave generation and wave-body interactions using a 3-D MEL model. In: Procedings of the 21st Symposium on Naval Hydro, Trondheim, Norway; 1997. P. 93-109.

[2] Ferrant P. Fully nonlinear interactions of long-crested wave packets with a three dimensional body. In: Procedings of the 22nd Symposium on Naval Hydro, Washington, USA; 1999. P. 403-15.

[3] Ferrant P, Le Touzé D, Pelletier K. Nonlinear time-domain models for irregular wave diffraction about offshore structures. Int J Numer Meth Fluids 2003; 43: 1257-77.

[4] Ducrozet G, Engsig-Karup AP, Bingham HR, Ferrant P. A non-linear wave decomposition model for efficient wave-structure interaction. Part A: Formulation, validations and analysis. J Comput Phys 2014; 257(15): $863-883$.

[5] Zhou BZ, Wu GX, Teng B. Fully nonlinear wave interaction with freely floating non-wall-sided structures. Eng Anal Bound Elem 2015; 50: 117-132.

[6] Zhou BZ, Wu GX. Resonance of a tension leg platform exited by third-harmonic force in nonlinear regular waves. Phil Trans R Soc A 2015; 373: 20140105.

[7] Ma QW, Wu GX, Eatock Taylor R. Finite element simulation of fully non-linear interaction between vertical cylinders and steep waves. Part 2: Numerical results and validation. Int J Numer Meth Fluids 2001; 36: 287-308.

[8] $\mathrm{Wu} \mathrm{GX,} \mathrm{Hu} \mathrm{ZZ.} \mathrm{Simulation} \mathrm{of} \mathrm{nonlinear} \mathrm{interactions} \mathrm{between} \mathrm{waves} \mathrm{and} \mathrm{floating} \mathrm{bodies} \mathrm{through} \mathrm{a}$ finite-element-based numerical tank. Proc R Soc Lond A 2004; 460: 2797-817. 
[9] Wang CZ, Wu GX. An unstructured-mesh based finite element simulation of wave interactions with non-wall-sided bodies. J Fluid Struct 2006; 22:441-461.

[10] Ma QW, Yan S. QALE-FEM numerical modelling of non-linear interaction between 3D moored floating bodies and steep waves. Int J Numer Numer Meth Eng 2009; 78: 713-756.

[11] Liu YM, Xue M, Yue DKP. Computations of fully nonlinear three dimensional wave-wave and wave-body interactions. Part 2: Nonlinear waves and forces on a body. J Fluid Mech 2001; 438: 41-46.

[12] Bai W, Eatock Taylor R. Fully nonlinear simulation of wave interaction with fixed and floating flares structures. Ocean Eng 2009; 36: 223-36.

[13] Bai W, Feng X, Eatock Taylor R, Ang KK. Fully nonlinear analysis of near-trapping phenomenon around an array of cylinders. Appl Ocean Res 2014; 44:71-81.

[14] Yan HM, Liu YM. An efficient high-order boundary element method for nonlinear wave-wave and wave-body interactions. J Comput Phys 2011; 230(2):402-424.

[15] Scolan YM, Le Boulluec M, Chen XB, Deleuil G, Ferrant P, Malenica S, Molin B. Some results from numerical and experimental investigations on the high frequency responses of offshore structures. Proc. of BOSS'97, Delft, Netherland,1997.

[16] Chaplin JR, Rainey RCT, Yemm RW. Ringing of a vertical cylinder in waves. J. Fluid Mech 1997; $350: 119-147$.

[17] Stansberg CT, Huse E, Krogstad JR, Lehn E. Experimental Study of Non-Linear Loads on Vertical Cylinders in Steep Random Waves. Proc. 5th Int. Offshore and Polar Eng. Conf. International Society of Offshore and Polar Engineers. Hague, Netherlands, 1995.

[18] Korteweg DJ, De Vries G. On the change of form of long waves advancing in a rectangular canal and on a new type of long stationary wave. Phil Mag 1895; 39(5): 422-443.

[19] Laitone EV. The second approximation to cnoidal and solitary waves. J. Fluid Mech 1960; 9: 430-44.

[20] Grimshaw R. The solitary wave in water of variable depth. Part 3. J Fluid Mech 1971; 46: 611-22.

[21] Tanaka M. The stability of solitary waves. Phys Fluids 1986; 29(3): 650-55.

[22] Clamond D, Dutykh D. Fast accurate computation of the fully nonlinear solitary surface gravity waves. Comput Fluids 2013; 84: 35-8.

[23] Maxworthy T. Experiments on collisions between solitary waves. J Fluid Mech 1976; 76: 177-85.

[24] Chen YS, Yeh H. Laboratory experiments on counter-propagating collisions of solitary waves. Part 1 . Wave interactions. J Fluid Mech 2014; 749: 577-96. 
[25] Cooker MJ, Weidman PD, Bale DS. Reflection of a high-amplitude solitary wave at a vertical wall. J Fluid Mech 1997; 342:141-158.

[26] Craig W, Guyenne P, Hammack J, Henderson D, Sulem C. Solitary wave interactions. Phys Fluids 2006; 18: $1-25$.

[27] Chambarel J, Kharof C, Touboul J. Head-on collision of two solitary waves and residual falling jet formation. Nonlinear Proc Geoph 2009; 16: 111-22.

[28] Sun JL, Wang CZ, Wu GX, Khoo BC. Fully nonlinear simulations of interactions between solitary waves and structures based on the finite element method. Ocean Eng. 2015; 108: 202-15.

[29] Su CH, Mirie RM. On head-on collisions between two solitary waves. J Fluid Mech 1980; 98: 509-25.

[30] Yates GT, Wang K. Solitary wave scattering by a vertical cylinder: experimental study. The Fourth International Offshore and Polar Engineering Conference. International Society of Offshore and Polar Engineers, 1994.

[31] Mo W, Irschik K, Oumeraci H, Liu PF. A 3D numerical model for computing non-breaking wave forces on slender piles. J Eng Math 2007; 58 (1-4), 19-30.

[32] Zhao M, Cheng L, Teng B. Numerical simulation of solitary wave scattering by a circular cylinder array, Ocean Eng 2007; 34(3-4): 489-99.

[33] Zhong Z, Wang KH. Time-accurate stabilized finite-element model for weakly nonlinear and weakly dispersive water waves, Int J Numer Meth Fluids 2008; 57(6): 715-44.

[34] Zhong Z, Wang K H. Modeling fully nonlinear shallow-water waves and their interactions with cylindrical structures. Comput Fluids 2009; 38(5): 1018-25.

[35] Cao HJ, Wan DC. RANS-VOF solver for solitary wave run-up on a circular cylinder. China Ocean Eng 2015; 29(2): 183-96.

[36] Isaacson MDSQ. Nonlinear-wave effects on fixed and floating bodies. J Fluid Mech 1982; 120: 267-81.

[37] Wu GX, Eatock Taylor R. The coupled finite element and boundary element analysis of nonlinear interactions between waves and bodies. Ocean Eng 2003; 30: 387-400.

[38] Thornton ST, Marion JB. Classical Dynamics of Particles and Systems, fifth edition. USA, 2003; 440-447.

[39] Li HB, Han GM, Mang HA. A new method for evaluating singular integrals in stress analysis of solids by the direct boundary element method. Int J Numer Numer Meth Eng 1985; 211: 2071-2075.

[40] Carnahan B, Luther HA, Wilkes JO. Applied Numerical Methods. Krieger Pub Co. Malabar, Florida, 1990, 363. 